\title{
Annealing-induced magnetic moments detected by spin precession measurements in epitaxial graphene on $\mathrm{SiC}$
}

\author{
Bastian Birkner, ${ }^{1, *}$ Daniel Pachniowski, ${ }^{1}$ Andreas Sandner, ${ }^{1}$ Markus Ostler, ${ }^{2}$ Thomas Seyller,,${ }^{2, \dagger}$ Jaroslav Fabian, ${ }^{3}$ \\ Mariusz Ciorga, ${ }^{1}$ Dieter Weiss, ${ }^{1}$ and Jonathan Eroms ${ }^{1}$ \\ ${ }^{1}$ Institute of Experimental and Applied Physics, University of Regensburg, 93040 Regensburg, Germany \\ ${ }^{2}$ Lehrstuhl für Technische Physik, University of Erlangen-Nürnberg, 91058 Erlangen, Germany \\ ${ }^{3}$ Institute of Theoretical Physics, University of Regensburg, 93040 Regensburg, Germany \\ (Received 11 October 2012; revised manuscript received 21 December 2012; published 14 February 2013)
}

\begin{abstract}
We present results of nonlocal and three-terminal (3T) spin precession measurements on spin injection devices fabricated on epitaxial graphene on $\mathrm{SiC}$. The measurements were performed before and after an annealing step at $150{ }^{\circ} \mathrm{C}$ for 15 minutes in vacuum. The values of spin relaxation length $L_{s}$ and spin relaxation time $\tau_{s}$ obtained after annealing are reduced by a factor 2 and 4, respectively, compared to those before annealing. An apparent discrepancy between spin diffusion constant $D_{s}$ and charge diffusion constant $D_{c}$ can be resolved by investigating the temperature dependence of the $g$ factor, which is consistent with a model for paramagnetic magnetic moments.
\end{abstract}

DOI: 10.1103/PhysRevB.87.081405

PACS number(s): 72.25.-b, 72.80.Vp, 85.75.-d

Apart from its prospects for electronic devices ${ }^{1,2}$ singlelayer graphene (SLG) is also a very promising candidate in the field of spintronics because it is expected that spin information can be passed in graphene over long distances ${ }^{3}$ due to the weak spin-orbit coupling and low hyperfine interaction. ${ }^{4} \mathrm{Up}$ to now, however, the measured spin lifetimes in exfoliated SLG $(0.5 \mathrm{~ns}$ at $\mathrm{RT}^{5} \approx 1 \mathrm{~ns}$ at $\left.4 \mathrm{~K}^{6}\right)$ and also in bilayer graphene $(\approx 2 \mathrm{~ns}$ at $\left.\mathrm{RT}^{7}\right)$ on $\mathrm{SiO}_{2}$ are still one order of magnitude smaller than in conventional semiconductor heterostructures. Even if the mobility $\mu$ for graphene on $\mathrm{SiO}_{2}$ is modified by, e.g., ligandbound nanoparticles ${ }^{8}\left(2700-12000 \mathrm{~cm}^{2} / \mathrm{V} \mathrm{s}\right)$ or by using highquality suspended graphene devices ${ }^{9}\left(\mu>100000 \mathrm{~cm}^{2} / \mathrm{V} \mathrm{s}\right)$, measured spin lifetimes are below 2 ns. Similar values of $\tau_{s}$, slightly over $2 \mathrm{~ns}$, were also reported for graphene epitaxially grown on a semi-insulating silicon carbide ( $\mathrm{SiC}$ ) substrate $^{10,11}$ using a direct nonlocal measurement ${ }^{12}$ while a huge $\tau_{s}$ was obtained by an indirect ${ }^{13}$ method. In Ref. 12 fitting the Hanle curves with a $g$ factor of 2 leads to a drastic difference between charge $\left(D_{c}\right)$ and spin diffusion constant $\left(D_{s}\right)$. Later the data were reinterpreted in a model employing a modified $g$ factor. ${ }^{14}$ McCreary et al. ${ }^{15}$ studied the influence of artificially created paramagnetic moments on spin transport in graphene, and introduced an effective exchange field model leading to an enhanced $g$ factor. This variety of different results both at room and low temperature motivates further experiments on epitaxial graphene to understand the spin relaxation mechanism in order to control the spin information for future spintronic devices.

Here we also use epitaxial graphene grown on the Si face of $\mathrm{SiC}$ and present nonlocal and three-terminal ${ }^{16}$ spin precession measurements. The latter probes the spin accumulation ${ }^{17}$ directly underneath the injector electrode induced electrically by a spin-polarized current. We compare the results before and after an annealing step and observe that our measurements after annealing can be well explained with an enhanced $g$ factor assuming that $D_{c}$ and $D_{s}$ are equal. As the temperature dependence of this increased $g$ factor shows a clear $1 / T$ (paramagnetic) behavior, we believe that annealing creates local magnetic moments which influence the spin transport properties.
Figure 1(a) shows a sketch of the applied measurement methods, Fig. 1(b) a SEM picture of the used epitaxial graphene spin injection device. The graphene stripes having a width of $W=30 \mu \mathrm{m}$ and a length of about $750 \mu \mathrm{m}$ are produced using a negative resist based electron beam lithography (EBL) step and oxygen plasma etching for $30 \mathrm{~s}$ (30 mTorr $\mathrm{O}_{2}, 50 \mathrm{~W}$ ). Afterwards a thin tunneling barrier $\left(\mathrm{AlO}_{x}\right)$ with a thickness of about $1 \mathrm{~nm}$ was produced by depositing $\mathrm{Al}$ atoms over the entire cooled sample $(180 \mathrm{~K})$ in a UHV system ( $p \approx 10^{-9} \mathrm{mbar}$ ) and subsequent oxidation in the load lock in pure oxygen atmosphere ( $p \approx 3 \times 10^{-2}$ mbar) at RT for 30 minutes. This $\mathrm{AlO}_{x}$ tunneling barrier with a contact resistance $R_{c} \geqslant 2 \mathrm{k} \Omega$ provides high spin injection efficiencies and reduces spin relaxation induced by the contacts. ${ }^{18}$ The ferromagnetic (FM) cobalt electrodes (Co $20 \mathrm{~nm}$ ) with a width of $200 \mathrm{~nm}$ (contact A) and $500 \mathrm{~nm}$ (contact B) and the nonmagnetic palladium contacts $(\mathrm{Pd} 80 \mathrm{~nm})$ were each patterned using a positive PMMA resist based EBL step. The evaporation is done via electron gun $(\mathrm{Co})$ and thermally $(\mathrm{Pd})$ at a base pressure of about $5 \times 10^{-7}$ mbar followed by a standard liftoff technique. The distance $L$ between the edges of the FM stripes is $2 \mu \mathrm{m}$. Finally the sample is glued into a chip carrier and the measurements are done using a standard dc setup in a Cryogenics He-4 cryostat $(T=1.6 \ldots 300 \mathrm{~K})$ equipped with a vector magnet $\left(B_{x, y, z}=-1 \ldots 1 \mathrm{~T}\right)$. The complete sample fabrication is done without applying a high temperature cleaning step.

In Fig. 2 typical Hanle curves in the nonlocal and threeterminal setup at $1.7 \mathrm{~K}$ are shown when using contact A as an injector. The FM stripes are magnetized in parallel configuration and the magnetic field $B_{z}$ is applied out of plane which leads to dephasing of the spin signal. In our convention, $R_{n l}$ is negative for parallel magnetization. The continuous curve for the nonlocal curve in Fig. 2(a) is the numerical fit with the solution of the following equation: ${ }^{19}$

$$
-R_{n l}=\frac{V_{n l}}{I}=\frac{P^{2} R_{s} L_{s}}{2 W} \int_{0}^{\infty} \frac{\cos \left(\omega_{L} t\right)}{\sqrt{4 \pi D_{s} t}} e^{\frac{-\left(x_{2}-x_{1}\right)^{2}}{4 \nu_{s} t}} e^{\frac{-t}{\tau_{s}}} d t,
$$

where $P$ is assumed to be the same for both FM stripes and $x_{1}, x_{2}$ are the points of injection and detection, respectively. 
(a)

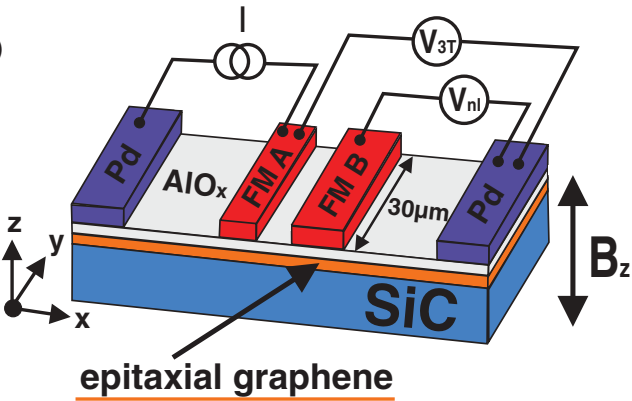

(b)

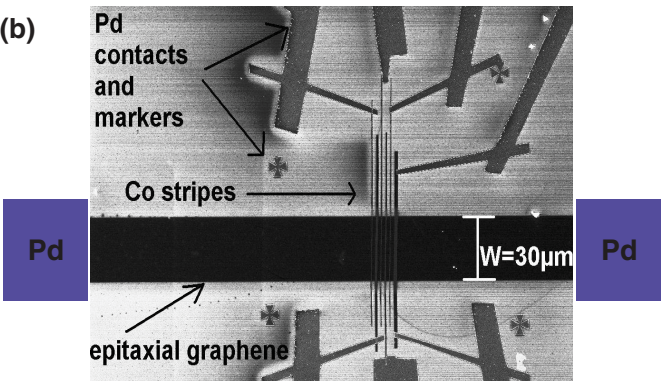

FIG. 1. (Color online) (a) Schematic drawing of the nonlocal and 3T measurement setup which is used for electrical spin injection and detection. The magnetic field $B_{z}$ is applied along the $z$ axis. (b) SEM picture of the epitaxial graphene spin valve device.

$P$ is the spin injection efficiency, $I$ the injection current, $\omega_{L}=$ $g \mu_{B} B_{z} / \hbar$ the Larmor frequency with the Landé factor $g, R_{s}$ the sheet resistance of graphene, $W$ the width of the graphene stripe, $D_{s}$ the spin diffusion constant, and finally $\tau_{s}$ and $L_{s}=$ $\sqrt{D_{s} \tau_{s}}$ the spin relaxation time and length, respectively. An influence of drift can be neglected due to the low bias current of $10 \mu \mathrm{A} .{ }^{17,20}$

The Hanle signal $R_{3 \mathrm{~T}}$ in $3 \mathrm{~T}$ configuration [Fig. 2(b)] can be fitted with the following Lorentzian ${ }^{16}$ curve:

$$
R_{3 \mathrm{~T}}=\frac{V_{3 \mathrm{~T}}}{I}=\frac{P^{2} R_{s} L_{s}}{2 W\left[1+\left(\omega_{L} \tau_{s}\right)^{2}\right]} .
$$

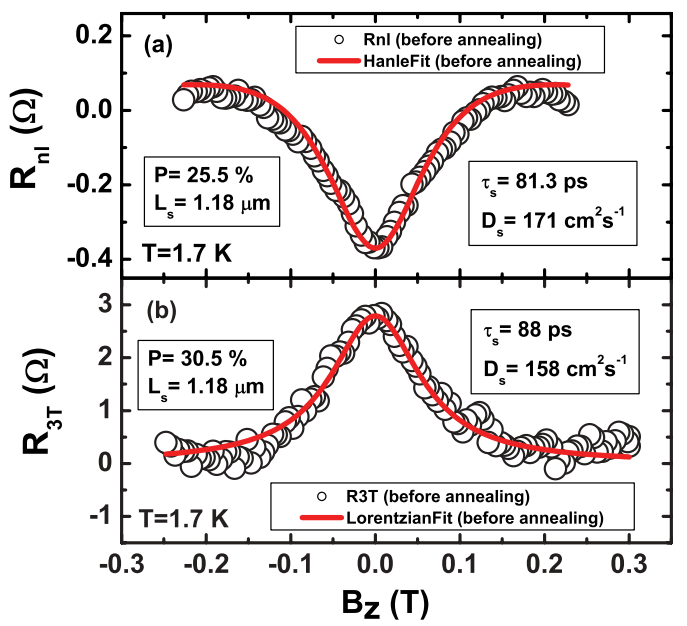

FIG. 2. (Color online) (a) Hanle spin precession measurement (background removed) with a dc current of $+10 \mu \mathrm{A}$ at $1.7 \mathrm{~K}$ and fit (continuous line) in nonlocal configuration. (b) $3 \mathrm{~T}$ measurement (background removed) and Lorentzian fit (continuous line). Both measurements are done before an annealing step.
We observe experimentally that $\tau_{s}$ from this fit coincides with $\tau_{s}$ obtained from the nonlocal measurement. ${ }^{21}$ As the amplitude of the nonlocal signal is determined by the product of $P^{2}$ and $L_{s}$ these parameters are not independent in the fitting procedure. Therefore $L_{s}=1.18 \mu \mathrm{m}$ is estimated assuming an exponential decay of the spin signal given by $R_{3 \mathrm{~T}}\left(B_{z}=0\right)$ at $L=0 \mu \mathrm{m}$ and $R_{n l}\left(B_{z}=0\right)$ at $L=2 \mu \mathrm{m}$. Now $P$ and $\tau_{s}$ are the free fitting parameters and $D_{s}$ can be calculated via $D_{s}=L_{s}^{2} / \tau_{s}$.

In Fig. 2 one can see that for both nonlocal and 3T spin precession measurements the results for $P, \tau_{s}$, and $D_{s}$ are almost identical. This agreement indicates that these signals originate from an induced spin accumulation into graphene. Slight differences especially in the spin injection efficiency $P$ can be explained by a small anisotropic magnetoresistance contribution of about $0.5 \Omega$ of the FM stripes to $R_{3 \mathrm{~T}}$ determined in reference measurements (not shown). This small deviation leads to an absolute error of $L_{s}$ of about $\Delta L_{s}=200 \mathrm{~nm}$. Fitting the nonlocal measurements, we obtain $\tau_{s}=81.3 \mathrm{ps}$ which is slightly smaller than in exfoliated SLG. ${ }^{3,18,22}$ The resulting spin diffusion constant $D_{s}=171 \mathrm{~cm}^{2} / \mathrm{s}$ is comparable to the charge diffusion constant $D_{c}=\frac{1}{2} l_{p} v_{F}=158 \mathrm{~cm}^{2} / \mathrm{s}$ extracted from a reference sample grown with identical parameters and also covered with $\mathrm{AlO}_{x}$ produced with the same processing steps as for the tunneling barriers. This similarity shows that the value of $D_{s}$ extracted from the Hanle fit is reliable. $l_{p}=\frac{\hbar}{e} \sqrt{n \pi} \mu$ is the mean-free path, $v_{F}=10^{6} \mathrm{~m} / \mathrm{s}$ is the Fermi velocity in graphene, $n=5.9 \times 10^{12} \mathrm{~cm}^{-2}$ is the charge carrier density, and $\mu=1126 \mathrm{~cm}^{2} / \mathrm{V}$ s is the mobility of the reference sample.

In order to check whether annealing influences the charge transport properties and/or the induced spin accumulation, a postannealing step is done at $150{ }^{\circ} \mathrm{C}$ for 15 minutes in vacuum to avoid intercalation of hydrogen ${ }^{23,24}$ via forming gas. Then we repeat the same spin precession measurements as before and interestingly we observe that $\tau_{s}$ increases whereas $D_{s}$ is decreased by almost a factor of 5 if we assume the same $g$ factor as before annealing $\left(g=g_{0}=2\right)$. For the configuration with contact $\mathrm{B}$ as an injector we observe an even bigger decrease of $D_{s}$ by a factor of about 10 (Table I) which can be explained by an inhomogeneity of $R_{s}$ after $\mathrm{AlO}_{x}$ deposition also observed in the reference sample. At this point we conclude that annealing affects the spin transport properties and we observe the same apparent reduction of $D_{s}$ as in Ref. 12, where a high temperature annealing step was included in the sample preparation procedure. $L_{s}=594 \mathrm{~nm}$ is reduced by a factor of 2 after annealing and is again extracted from the exponential decay of the spin signal at the injection point $\left[R_{3 \mathrm{~T}}(0)\right]$ and at a distance $L=2 \mu \mathrm{m}\left[R_{n l}(0)\right]$. The fact that both $L_{s}$ and the 3T amplitude at zero magnetic field decrease by

TABLE I. $D_{s}\left(\mathrm{~cm}^{2} / \mathrm{s}\right), \tau_{s}(\mathrm{ps})$, and $g$ factor before and after annealing for injector contacts $\mathrm{A}$ and $\mathrm{B}$ at $T=1.7 \mathrm{~K}$. After annealing the measurements can also be fitted with an enhanced $g_{\text {eff }}>g_{0}$.

\begin{tabular}{lccccccccr}
\hline \hline \multicolumn{3}{l}{ Before annealing } & & \multicolumn{6}{c}{ After annealing } \\
\cline { 6 - 11 }$\tau_{s}$ & $D_{s}$ & $g_{0}$ & Injector & \multicolumn{1}{c}{$\tau_{s}$} & $D_{s}$ & $g_{0}$ & $\tau_{s}$ & $D_{s}$ & $g_{\text {eff }}$ \\
\hline 81.3 & 171 & 2 & $\mathrm{~A}$ & 95 & 37 & 2 & 22 & 160 & 8 \\
108 & 208 & 2 & $\mathrm{~B}$ & 165 & 21 & 2 & 22 & 160 & 11 \\
\hline \hline
\end{tabular}


almost the same factor indicates that the applied postannealing step also affects the induced spin accumulation underneath the injector electrode. As the spin transport sample did not allow us to determine the mobility and charge carrier density independently we also annealed the reference sample (covered with $\mathrm{AlO}_{x}$ ) under the same conditions as the spin transport sample. From low-field Hall measurements at $1.7 \mathrm{~K}$ we get an enhanced charge carrier density $n=8.4 \times 10^{12} \mathrm{~cm}^{-2}$ after annealing whereas the mobility just slightly increases to $\mu=1237 \mathrm{~cm}^{2} / \mathrm{V}$ s. We conclude now that a change in $R_{s}$ is mainly caused by a change in the charge carrier density. Following the results of the reference sample we ascribe the small sheet resistance increase from $R_{s}=1.5 \mathrm{k} \Omega$ before and $R_{s}=1.7 \mathrm{k} \Omega$ after annealing of the spin transport sample to a minute reduction in doping. The charge diffusion constant $D_{c}$ (and also $D_{s}$ ) is therefore slightly decreased from $171 \mathrm{~cm}^{2} / \mathrm{s}$ to $160 \mathrm{~cm}^{2} / \mathrm{s}\left(D_{c} \propto \sqrt{n}\right)$ for the spin transport sample. ${ }^{25}$ In conclusion, the minor decrease in $D_{c}$ due to the annealing step cannot explain the strong reduction of $D_{s}$ extracted from the Hanle fits. That means we have the following situation: $D_{c}^{\text {before }} \approx D_{c}^{\text {after }} \gg D_{s}^{\text {after }}$.

In an attempt to understand the discrepancy of $D_{c}$ and $D_{s}$ Maassen et al. ${ }^{12}$ first considered localized states in the electrically inert buffer layer ${ }^{26}$ (BL) which could provide hopping sites for the spins being able to change the spin transport properties but not the charge transport properties. The difference in $D_{s}$ and $D_{c}$ was also recently discussed by McCreary et al. ${ }^{15}$ They assume a formation of local magnetic moments by Ar sputtering or from hydrogen adatoms on exfoliated graphene samples which provide an enhanced magnetic field for the diffusing spins, which can be modeled by an effective $g$ factor. Maassen et al. ${ }^{14}$ reinterpreted their experiments $^{12}$ using a model of localized states, where the effective Larmor frequency is increased in the limit of strong coupling, which again can be expressed by an enhanced $g$ factor and then allows setting $D_{c}=D_{s}$.

For this reason we also fit our nonlocal and $3 \mathrm{~T}$ data after annealing, treating the $g$ factor in the Larmor frequency [Eqs. (1) and (2)] as a free parameter, and assuming $D_{c}^{\text {after }}=$ $D_{s}^{\text {after }}=160 \mathrm{~cm}^{2} / \mathrm{s}$. Figure 3 shows that our data can also be well fitted with an enhanced $g$ factor of 8 in both the nonlocal and in the 3T setup. The oscillations observed in the Hanle curve [Fig. 3(a)] at higher magnetic fields are phasecoherent contributions and vanish at higher temperatures. If we summarize our experimental findings so far (Table I) we can conclude that our measurements after annealing can be explained either by $D_{c}^{\text {after }} \neq D_{s}^{\text {after }}$ or by an effective Landé factor $g_{\text {eff }}>2$ as both models reproduce the data equally well since Eqs. (1) and (2) contain the $g$ factor implicitly in the Larmor frequency $\omega_{L}$ and are invariant under a rescaling of $g$, $\tau_{s}$, and $D_{s} .{ }^{14}$

To determine which model (hopping or magnetic moments) is appropriate in our situation we study the temperature dependence of spin transport. We observe that the enhanced effective $g$ factor, as well as the amplitude of the spin signal, decrease with increasing temperature. From the $T$ dependence of the reference sample and the $T$ dependence of $R_{s}$ of the spin transport sample after annealing we conclude that $D_{c}$ is weakly influenced by the temperature. This was also included in the Hanle fits (Fig. 4).

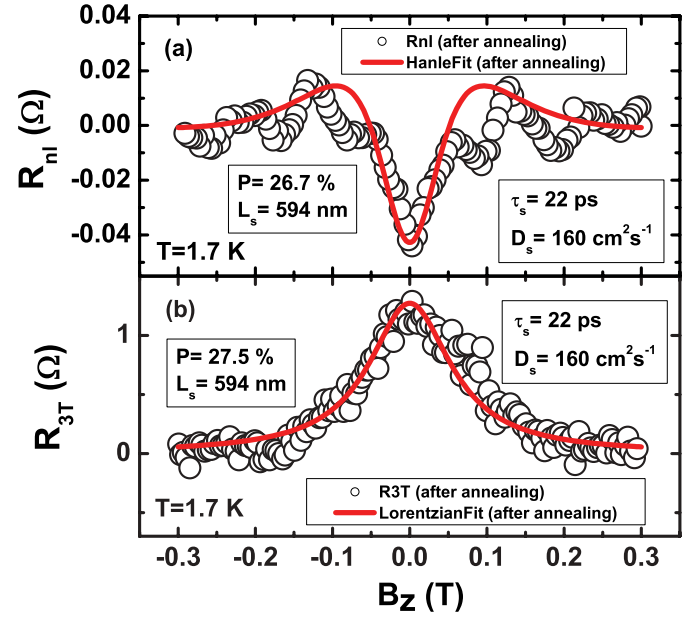

FIG. 3. (Color online) (a) Hanle spin precession measurement (background removed) at $1.7 \mathrm{~K}$ and fit (continuous line) in nonlocal configuration. (b) $3 \mathrm{~T}$ measurement (background removed) and Lorentzian fit (continuous line). Both measurements are done after an annealing step. The fittings are done treating the $g$ factor as a free parameter.

If $g_{\text {eff }}$ originates from magnetic moments then its temperature dependence can be described by the following equation: ${ }^{15}$

$$
g_{\mathrm{eff}}(T)=g_{0}+\frac{g_{0} \eta_{M} A_{\mathrm{ex}}}{k_{B} T} .
$$

This is the low-field approximation of the Brillouin function of a spin-1/2 paramagnetic material. $A_{\mathrm{ex}}$ is the strength of the exchange coupling, $\eta_{M}$ represents the filling density of the magnetic moments, $g_{0}=2$ is the $g$ factor for free electrons, and $k_{B}$ is the Boltzmann constant. As is seen in Fig. 5 the measured temperature dependence of $g_{\text {eff }}$ is well described by Eq. (3). This temperature dependence is compatible with the effective exchange field model proposed by McCreary et al. ${ }^{15}$ which describes the enhancement of the magnetic field felt by the diffusing spins due to localized paramagnetic moments. Maassen et al., ${ }^{14}$ on the other hand, interpret their data by hopping of the diffusing spins into localized states which leads to an apparent enhancement of the $g$ factor in the Hanle fit. In their work the increase of $g$ is most pronounced at room temperature, whereas in our case the maximum $g_{\text {eff }}$ is obtained at low temperature.

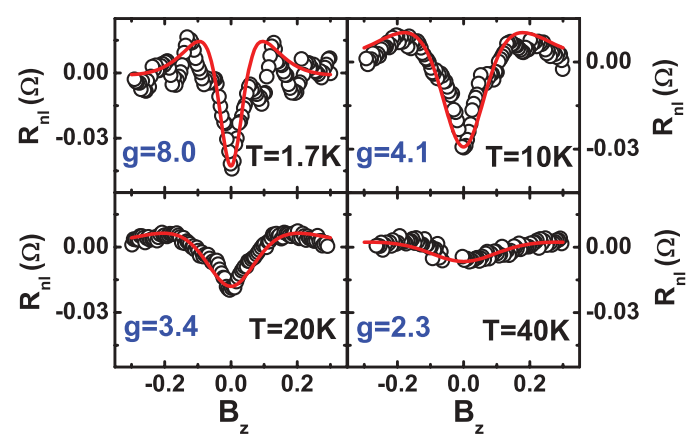

FIG. 4. (Color online) Temperature-dependent nonlocal Hanle measurements (background removed) with $g$ factor as further fitting parameter. 


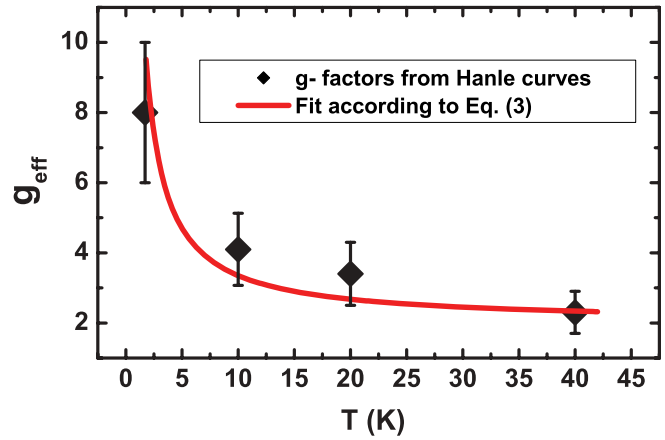

FIG. 5. (Color online) Temperature dependence of the $g$ factor. The continuous curve is the fit according to Eq. (3).

We therefore believe that postannealing creates an amount of randomly positioned magnetic moments resulting in an increased effective magnetic field $B_{\text {eff }}=B_{z}+B_{\text {ex }}$ composed of the applied out-of-plane magnetic field $B_{z}$ and of an exchange field $B_{\text {ex }}$ coming from the induced magnetic moments. ${ }^{27}$ This enhanced magnetic field can be modeled by an effective $g$ factor in the Larmor frequency $\omega_{L}$. As we nearly get the same Landé factor from the nonlocal Hanle and Lorentzian fit we cannot decide whether the magnetic moments are formed in the graphene/buffer layer transition or at the $\mathrm{AlO}_{x}$ /graphene interface. The difference between our experiment and the work of Maassen et al ${ }^{12}$ may be due to different annealing conditions.

As to the origin of the magnetic moments we assume that defects or vacancies ${ }^{28}$ which are already present in our epitaxial graphene are modified via the annealing step at
$150{ }^{\circ} \mathrm{C}$. One example could be step edges, which are known to occur frequently in epitaxial graphene on $\mathrm{SiC} .{ }^{11}$ This is supported by weak localization measurements on the reference sample, which yield a very short intervalley scattering length of $L_{i} \approx 40 \mathrm{~nm}$ and also by $\mathrm{THz}$ photocurrent experiments on the reference sample where photocurrents were detected in the bulk of the sample ${ }^{29,30}$ at normal incidence, which can only be explained by a lowering of the symmetry. ${ }^{31}$ Annealing then only changes the termination of the step edges, which influences their magnetic behavior.

In conclusion, an electrically induced spin imbalance from ferromagnetic Co stripes can be analyzed via spin precession measurements in both nonlocal and three-terminal configuration. By introducing a postannealing step, we observe that the spin relaxation length as well as the nonlocal and 3T Hanle amplitude decrease. Fitting of the nonlocal and 3T data after annealing shows an increase of the $g$ factor if spin and charge diffusion constants are assumed to be the same. The origin of the $g$-factor enhancement is local magnetic moments formed by annealing. The reduced spin lifetime and length support this assumption because local magnetic moments act as an additional spin scattering source. Finally, the temperature dependence shows clear evidence that paramagnetic moments are created as the effective $g$ factor scales with $1 / T$ with increasing temperature.

Support from the DFG within SFB 689 "Spin phenomena in reduced dimension" and SPP 1459 "Graphene" is gratefully acknowledged. We would like to thank F. Fromm, T. Maassen, S. Ganichev, and R. Kawakami for helpful discussions and T. Korn, F. Yaghobian, and C. Schüller for supporting Raman measurements. *bastian.birkner@physik.uni-regensburg.de

†Present address: Technische Universität Chemnitz, 09107 Chemnitz, Germany.

${ }^{1}$ A. H. Castro Neto, F. Guinea, N. M. R. Peres, K. S. Novoselov, and A. K. Geim, Rev. Mod. Phys. 81, 109 (2009).

${ }^{2}$ K. S. Novoselov, A. K. Geim, S. V. Morozov, D. Jiang, M. I. Katsnelson, I. V. Grigorieva, S. V. Dubonos, and A. A. Firsov, Nature (London) 438, 197 (2005).

${ }^{3}$ N. Tombros, C. Józsa, M. Popinciuc, H. T. Jonkman, and Bart J. van Wees, Nature (London) 448, 571 (2007).

${ }^{4}$ D. Huertas-Hernando, F. Guinea, and A. Brataas, Phys. Rev. B 74, 155426 (2006).

${ }^{5}$ W. Han, K. Pi, K. M. McCreary, Y. Li, J. J. I. Wong, A. G. Swartz, and R. K. Kawakami, Phys. Rev. Lett. 105, 167202 (2010).

${ }^{6}$ W. Han and R. K. Kawakami, Phys. Rev. Lett. 107, 047207 (2011). ${ }^{7}$ T.-Y. Yang, J. Balakrishnan, F. Volmer, A. Avsar, M. Jaiswal, J. Samm, S. R. Ali, A. Pachoud, M. Zeng, M. Popinciuc, G. Güntherodt, B. Beschoten, and B. Özyilmaz, Phys. Rev. Lett. 107, 047206 (2011).

${ }^{8}$ W. Han, J.-R. Chen, D. Wang, K. M. McCreary, H. Wen, A. G. Swartz, J. Shi, and R. Kawakami, Nano Lett. 12, 3443 (2012).

${ }^{9}$ M. H. D. Guimarães, A. Veligura, P. J. Zomer, T. Maassen, I. J. Vera-Marun, N. Tombros, and B. J. van Wees, Nano Lett. 12, 3512 (2012).
${ }^{10}$ P. N. First, W. A. de Heer, T. Seyller, C. Berger, J. A. Stroscio, and J.-S. Moon, MRS Bull. 35, 296 (2010).

${ }^{11}$ K. V. Emtsev, A. Bostwick, K. Horn, J. Jobst, G. L. Kellogg, L. Ley, J. L. McChesney, T. Ohta, S. A. Reshanov, J. Röhrl, E. Rotenberg, A. K. Schmid, D. Waldmann, H. B. Weber, and T. Seyller, Nat. Mater. 8, 203 (2009).

${ }^{12}$ T. Maassen, J. J. van den Berg, N. IJbema, F. Fromm, T. Seyller, R. Yakimova, and B. J. van Wees, Nano Lett. 12, 1498 (2012).

${ }^{13}$ B. Dlubak, M.-B. Martin, C. Deranlot, B. Servet, S. Xavier, R. Mattana, M. Sprinkle, C. Berger, W. A. De Heer, F. Petroff, A. Anane, P. Seneor, and A. Fert, Nat. Phys. 8, 557 (2012).

${ }^{14}$ T. Maassen, J. J. van den Berg, E. H. Huisman, H. Dijkstra, F. Fromm, T. Seyller, and B. J. van Wees, Phys. Rev. Lett. 110, 067209 (2013)

${ }^{15}$ K. M. McCreary, A. G. Swartz, W. Han, J. Fabian, and R. K. Kawakami, Phys. Rev. Lett. 109, 186604 (2012).

${ }^{16}$ S. P. Dash, S. Sharma, R. S. Patel, M. P. de Jong, and R. Jansen, Nature (London) 462, 491 (2009).

${ }^{17}$ J. Fabian, A. Matos-Abiague, C. Ertler, P. Stano, and I. Zutic, Acta Physica Slovaca 57, 565 (2007).

${ }^{18}$ M. Popinciuc, C. Józsa, P. J. Zomer, N. Tombros, A. Veligura, H. T. Jonkman, and B. J. van Wees, Phys. Rev. B 80, 214427 (2009). 
${ }^{19}$ M. Ciorga, A. Einwanger, U. Wurstbauer, D. Schuh, W. Wegscheider, and D. Weiss, Phys. Rev. B 79, 165321 (2009).

${ }^{20}$ M. Kameno, Y. Ando, E. Shikoh, T. Shinjo, T. Sasaki, T. Oikawa, Y. Suzuki, T. Suzuki, and M. Shiraishi, Appl. Phys. Lett. 101, 122413 (2012).

${ }^{21}$ Strictly speaking, the Lorentzian is only obtained for contacts much wider than the spin relaxation length. In our case, this may lead to an underestimated $\tau_{s}$ by a factor of 2 .

${ }^{22}$ W. Han and R. K. Kawakami, Phys. Rev. Lett. 107, 047207 (2011).

${ }^{23}$ C. Riedl, C. Coletti, T. Iwasaki, A. A. Zakharov, and U. Starke, Phys. Rev. Lett. 103, 246804 (2009).

${ }^{24}$ F. Speck, J. Jobst, F. Fromm, M. Ostler, D. Waldmann, M. Hundhausen, H. B. Weber, and T. Seyller, Appl. Phys. Lett. 99, 122106 (2011).

${ }^{25}$ See Supplemental Material at http://link.aps.org/supplemental/ 10.1103/PhysRevB.87.081405 for control measurements and more details on the effective exchange field model.
${ }^{26}$ K. V. Emtsev, F. Speck, T. Seyller, L. Ley, and J. D. Riley, Phys. Rev. B 77, 155303 (2008).

${ }^{27}$ For further discussion regarding the dip feature around $B=$ 0 when $B$ is applied along the ferromagnetic stripes see Ref. 25.

${ }^{28}$ O. V. Yazyev and L. Helm, Phys. Rev. B 75, 125408 (2007).

${ }^{29}$ J. Karch, C. Drexler, P. Olbrich, M. Fehrenbacher, M. Hirmer, M. M. Glazov, S. A. Tarasenko, E. L. Ivchenko, B. Birkner, J. Eroms, D. Weiss, R. Yakimova, S. Lara-Avila, S. Kubatkin, M. Ostler, T. Seyller, and S. D. Ganichev, Phys. Rev. Lett. 107, 276601 (2011)

${ }^{30}$ J. Karch, P. Olbrich, M. Schmalzbauer, C. Zoth, C. Brinsteiner, M. Fehrenbacher, U. Wurstbauer, M. M. Glazov, S. A. Tarasenko, E. L. Ivchenko, D. Weiss, J. Eroms, R. Yakimova, S. Lara-Avila, S. Kubatkin, and S. D. Ganichev, Phys. Rev. Lett. 105, 227402 (2010).

${ }^{31}$ S. D. Ganichev (private communication). 